\title{
Process Optimization and Thin Film Characterization of ZnO Layer for Sensor Applications
}

\author{
Kamaljeet Singh ${ }^{1}$, A V Nirmal ${ }^{1}$, Lokesh Rana $^{2}$, Monika Tomar ${ }^{2}$, and Vinay Gupta ${ }^{2}$ \\ ${ }^{1}$ U R Rao Satellite Centre,Bangalore \\ ${ }^{2}$ Delhi University, Delhi \\ kamaljs@isac.gov.in
}

\begin{abstract}
ZnO}$ deposition parameters and layer characterizations of the film which is having optoelectronic and piezo electric properties can play an important role in the realization of reliable MEMS based sensor. Sensor performance is based on the material characteristics such as crystal orientation, thickness, surface morphology; dielectric constants which employs various characterization techniques and methodologies. The grain size, FWHM, reflectance and band gap of the deposited layers are also critical and varying with deposition parameters such as substrate and annealing temperature. Various trial runs to optimize the process parameter and subsequent characterization of the film under various conditions are detailed in this article. Further the effect of moisture on the film quality is plotted and comparative analysis with and without humidity is bought out.
\end{abstract}

Index Terms: Thin film, zinc oxide, characterization, sensors

\section{Introduction}

Zinc oxide is having excellent piezoelectric as well as dielectric properties. The sensors based on piezo electric film are prominently employed for the realization of the acoustic sensors [1], gas sensors[2], microphones, ultrasonic transducers, FBAR device which finds applications in space, defence and commercial domain [3]. It is also a direct bandgap material and can also be employed for SAW devices leading to low loss and power [4]. Thin film layer deposition of $\mathrm{ZnO}$ depends upon multiple factors such as base substrate temperature, deposition methodology, gas composition, target size etc. Various other parameters such as annealing, RF power, deposition rate also affects the film characteristics such as uniformity, surface roughness, grain size, thickness etc.

The $\mathrm{ZnO}$ based sensor [5] realization employs various process steps such as diffusion, wet/dry etching, lithography, metallization in the process of fabrication flow. As the layer of $\mathrm{ZnO}$ encounters various temperature excursions along with chemical exposures, so cleaning methodology at various steps to remove polymers needs to be selected accordingly to avoid process contamination [6].

In this article thin film deposition of $\mathrm{ZnO}$ on silicon with the optimized process parameters and characterization results of the same are presented. It is reported that Si substrate makes it possible to have crystalline structure when films deposited near to pure zinc oxide. The deposited film is characterized for the grain size, thickness, band gap, reflectance, FWHM using various characterization methodologies [7]. The optimized process parameters are employed to realize the zinc oxide film and humidity trials on the same are carried out to validate the process ruggedness which is needed for reliable sensor operations. Earlier reported experimentation carried out without substrate temperature [8] but in this article the effect of the substrate temperature on the film properties are presented. This article details structural and electrical characterization of the film detailing role of various parameters to achieve repeatable piezoelectric sensor performance.

\section{Process parameters}

The process is carried out taking base layer of silicon with $\langle 100\rangle$ orientation after taking

Received: August $21^{\text {st }}, 2018$. Accepted: May $15^{\text {th }}, 2019$

DOI: 10.15676/ijeei.2019.11.2.3 
standard processes. The thin layer of $\mathrm{ZnO}$ deposition is carried out using the $\mathrm{RF}$ sputtering system with magnetron source and target size is chosen accordingly to achieve the uniform deposition [9]. Various parameters associated with thin layer deposition are:

- Gas composition

- Power

- Growth temperature

- Deposition rate

- Pressure

- Target-substrate distance

Various deposition techniques such as sputtering, sol-gel, pulse laser are employed whereas puttering is preferred due to good adhesion, large area deposition, requirement of low substrate temperature [10]. The deposition of the thin layer using sputtering should employ pre-sputtering step consisting of the pre-sputtering for small time in Ar atmosphere before actual deposition so as to remove contamination. This ensures the stability of the system and while keeping intact the optimum condition. The target to substrate distance is kept $>10 \mathrm{~cm}$ and deposition power > 100W. In present analysis, $\mathrm{Ar}$ and $\mathrm{O}_{2}$ ratio of 60:40 is taken and trial runs are conducted using bare silicon (4" and 6" dia) with vacuum level of $10^{-3}-10^{-5}$. The targeted thickness of $\mathrm{ZnO}$ is taken $\sim$ um for the analysis with the deposition rate kept between $0.2-0.8 \mathrm{umh}^{-1}$. The purity of the zinc target in the present study is taken as $99.99 \%$ and the pressure is kept below 20mTorr. Substrate temperature plays role in the crystallinity of the deposited film which is verified by experimentation and detailed in subsequent section. The present study is based on the optimization of the temperature for depositing the layer of $\mathrm{ZnO}$ achieving c-axis orientation.

\section{Structural Analysis}

Various measurement and characterization techniques can be employed for the film analysis. XRD,SEM,AFM and FTIR are employed for the morphological study of the deposited film. Table 1 shows the techniques and the corresponding properties are shown:

Table 1. Characterization techniques and parameters

$\begin{array}{lll}1 & \text { C-axis orientation } & \text { XRD } \\ 2 & \text { Energy bandgap } & \text { Optical absorption/ STM (STS) } \\ 3 & \text { FWHM } & \text { XRD } \\ 4 & \text { Grain Size } & \text { XRD, AFM, SEM } \\ 5 & \text { Adhesion } & \text { SEM } \\ 6 & \text { Thickness/ Height } & \text { AFM, SEM, Profilometer, Reflectance } \\ 7 & \text { Impurity/Contamination } & \text { EDAX, XPS, XRD } \\ 8 & \text { Zn/O ratio (content) } & \text { EDAX, XPS }\end{array}$

The dies are subjected to moisture exposure and humidity conditions of $95 \%$ are kept in the chamber. The FTIR response of the same is shown in the Figure 1. 

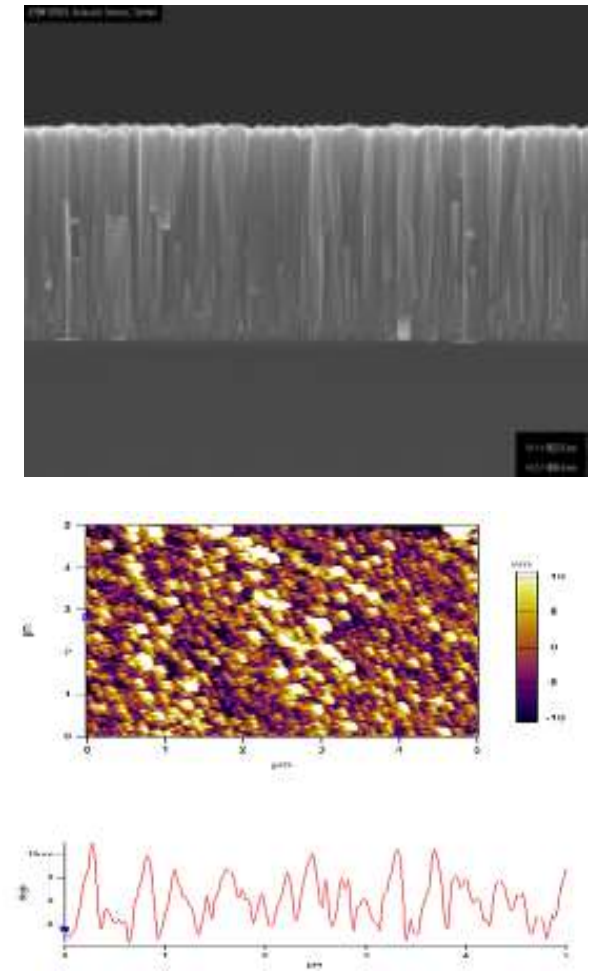

Figure 1. SEM image and surface roughness of the deposited $\mathrm{ZnO}$ layer

EDAX analysis for the composition of the film (table-2) shows the composition of the film.

Table 2. Film Composition

$\begin{array}{lcc}\text { Element } & \text { Weight\% } & \text { Atomic\% } \\ \mathrm{C} \mathrm{K}_{\text {edge }} & 2.00 & 6.24 \\ \mathrm{O} \mathrm{K} \text { edge } & 21.15 & 49.62 \\ \text { Zn Ledge } & 76.86 & 44.14 \\ \text { Total 100.00 } & & \end{array}$

The crystallographic orientation of the film can be found out using XRD and as $\mathrm{ZnO}$ is having hexagonal Wurtzite structure so for piezo-electric applications the crystallite should have c-axis perpendicular to the base substrate [11]. The intensity is determined with $2 \theta$ and peak at $\sim 34^{\circ}$ corresponds to the diffraction from (002) plane. The undesired intensity observed at (100) along with low intensity peak of (002) is attributed to the deterioration of the crystallinity of the films. The crystallinity is characterized using the $\mathrm{Cu}-\mathrm{K} \alpha$ radiation and the intensity is determined from $20^{\circ}$ to $40^{\circ}$ with small step size.

The full width half maximum (FWHM), as visible in the Figure 2, is due to contamination and the same disappeared after thorough cleaning of the chamber. 
Kamaljeet Singh, et al.

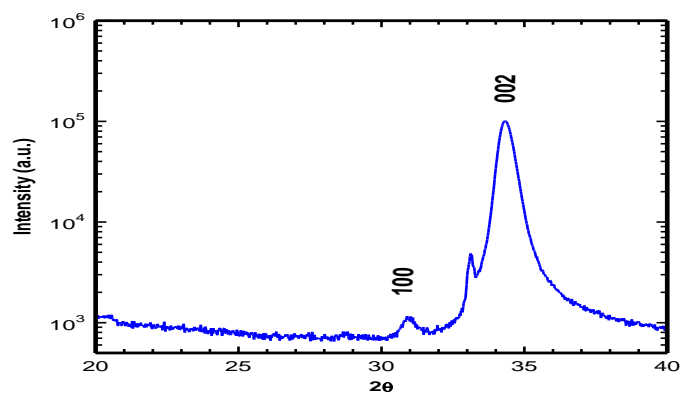

(a)

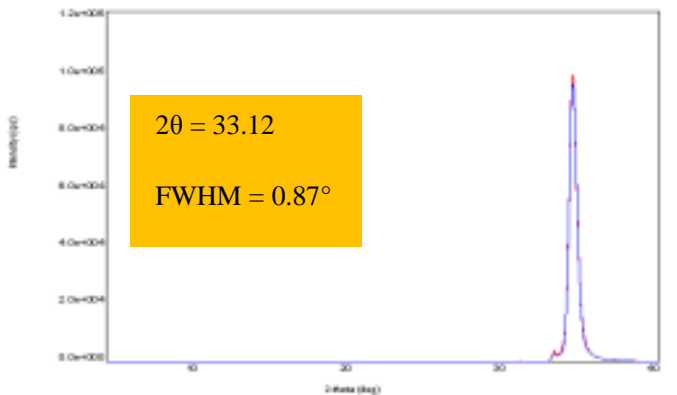

(b)

Figure 2. XRD plot of $\mathrm{ZnO}$ layer (a) standard (b) after trials
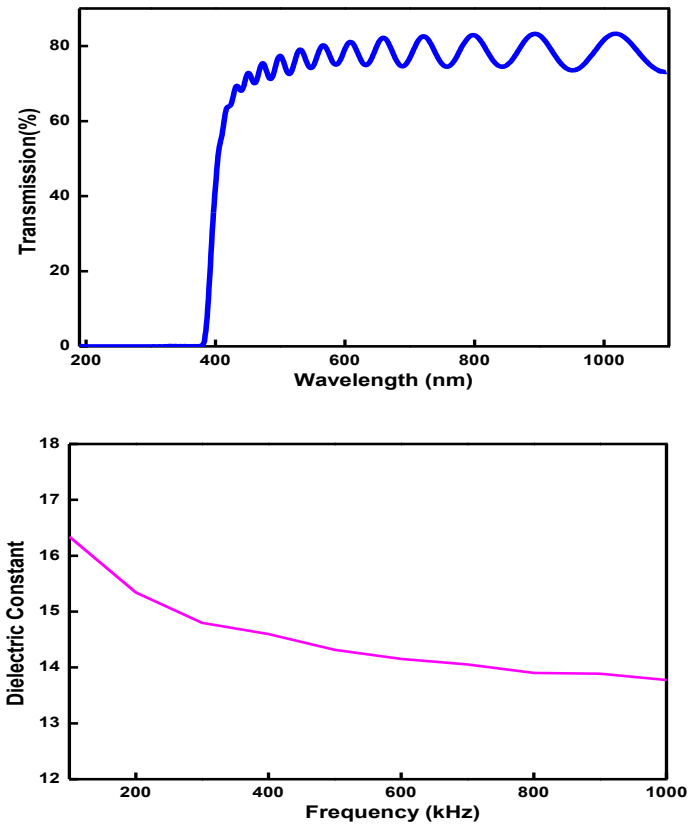

Figure 3. FTIR plot and dielectric constant measurement

The plot 2(b) shows highly c-axis oriented film and corresponding sharp peak which clearly indicates the role of the contamination on the film quality. The other measurement related with 
the dielectric constant, transmission and band gap is carried out and the plots are shown in Figure 3. The fourier transmission infrared (FTIR) plot indicates percentage of transmission behaviour with wavelength.

As shown above the band gap is slightly reduced whereas transmission peaks are also reduced with the enhanced substrate temperature. Various crystal parameters of the $\mathrm{ZnO}$ layer can be calculated using XRD data and calculated using the following standard equations:

$$
\begin{aligned}
& t \sim \frac{0.9 \lambda}{\beta \cos \theta} \\
& r \sim 0.625 \cdot \frac{\lambda}{\sin \theta} \\
& d \sim 0.5 \frac{\lambda}{\sin \theta} \\
& g \sim \frac{\beta}{\tan \theta}
\end{aligned}
$$

where $\lambda$ is the wavelength, $r$ is the inter-chain distance, $t$ is the grain size. The Table- 3 shows the calculated parameters of the $\mathrm{ZnO}$ film.

Table 3. Calculated parameters for the deposited thin film

\begin{tabular}{|l|l|l|}
\hline S.No & Parameters & Calculated \\
\hline 1 & Grain size $(\mathrm{t})$ & $30 \mathrm{~nm}$ \\
\hline 2 & Interchain distance $(\mathrm{r})$ & $1.7 \AA$ \\
\hline 3 & Interplanar distance $(\mathrm{d})$ & $2.61 \AA$ \\
\hline 4 & Distortion parameter (Lattice strain) $(\mathrm{g})$ & $0.86 \%$ \\
\hline
\end{tabular}

The calculated grain size is $\sim 30 \mathrm{~nm}$ which is validated by the AFM plot (Figure 4) which also shows the sizes are in range of $30-100 \mathrm{~nm}$. The size of the grain effects the reflectance and it decreases with the increase of grain size. The same can be measured using optiprobe to characterize contamination.

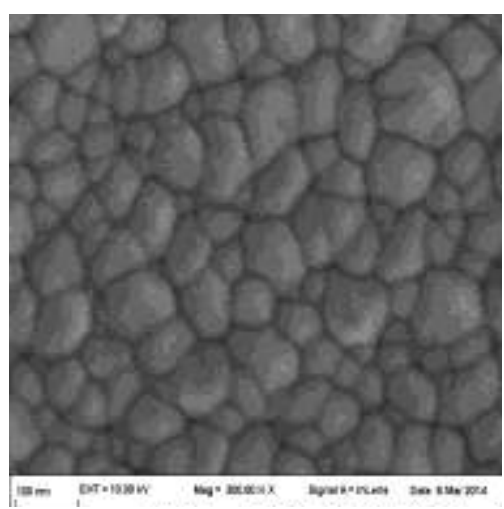

Figure 4. AFM plot of the deposited layer 
The above plot clearly indicates densely packed structure with uniform distributed grains.

\section{Effect of substrate temperature}

The effect of substrate temperature on the various parameters of $\mathrm{ZnO}$ is characterized and as shown below the difference in the properties with and without substrate temperature is shown in the Figure 5.
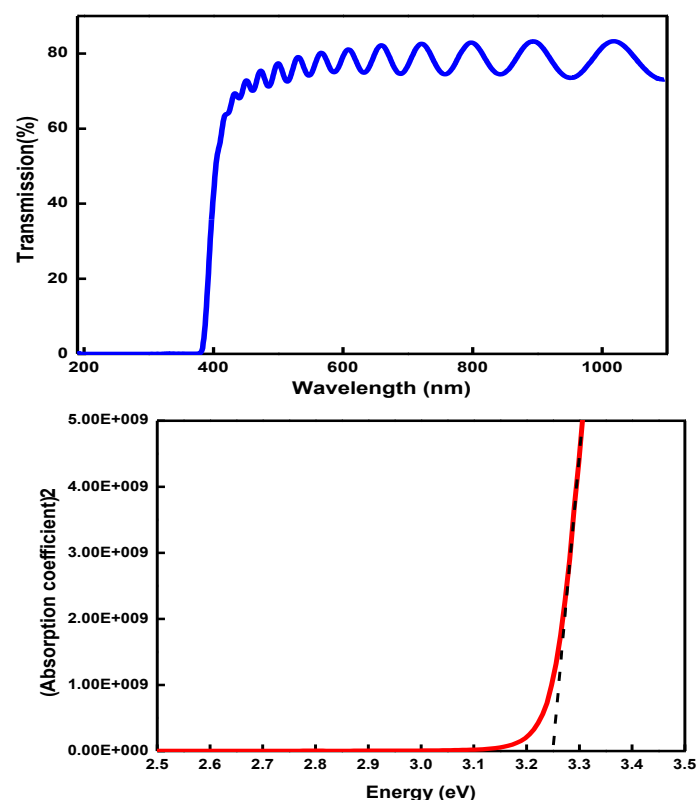

Figure 5. Transmission and absorption coefficient at room temperature

Various temperature options are considered and keeping device realization aspects the case study is carried out with temp $>250^{\circ} \mathrm{C}$ as shown in Figure 6.
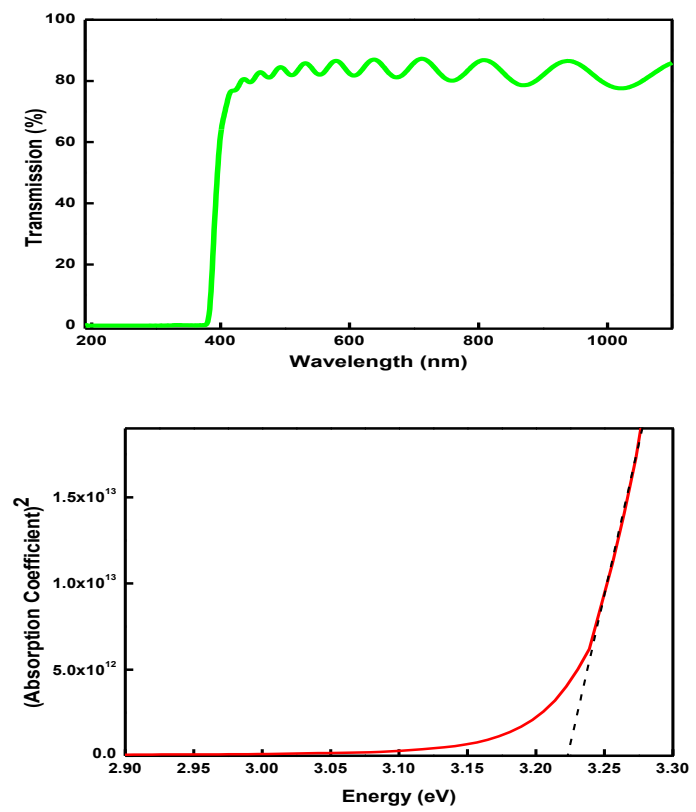

Figure 6. Transmission and absorption coefficient at $300^{\circ} \mathrm{C}$ 
This results in better response and further indicates better crystallinity due to annealing [12] the wafer in $\mathrm{O}_{2}$ environment. Thin film stress value also depends on the annealing duration and temperature settings which can be suitably explored keeping device application into considerations. [13]

\section{Humidity Response}

The effect of humidity on the sand-witch layer is analyzed as $\mathrm{ZnO}$ layer is embedded between oxide layer. PECVD deposited oxide layer is chosen and subjected to the humidity trials by analyzing the behaviour of the layer by keeping the thin layer deposited film in the RH chamber having $95 \%$ humidity. The effect of moisture is characterized with FTIR study which gives corresponding peak depending upon the electro-negativity of back bonds. Figure 7 shows the wavelength plot without and with humidity exposure on the deposited film.

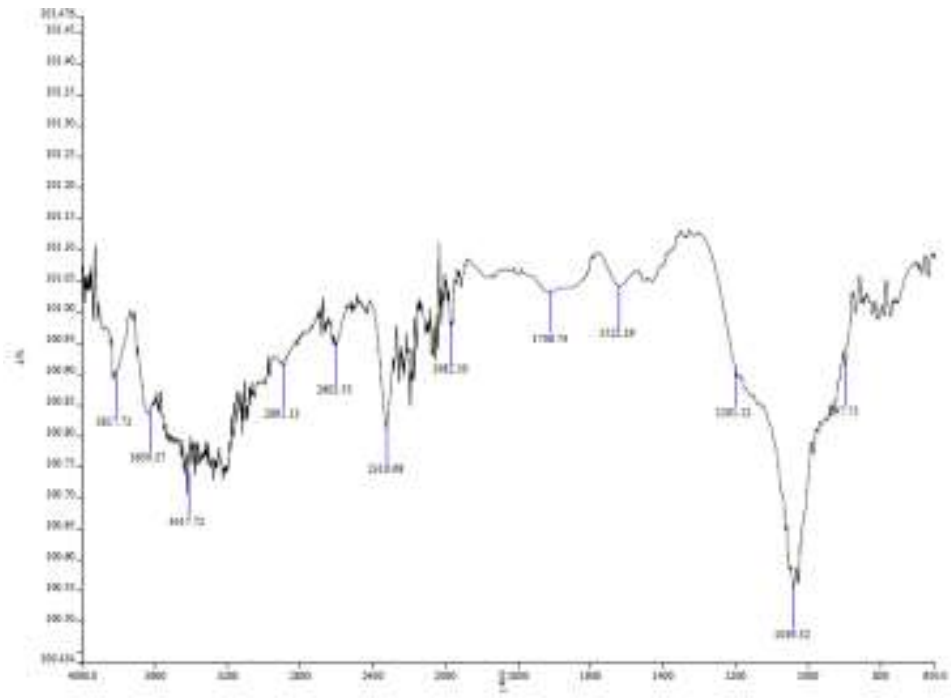

(a)

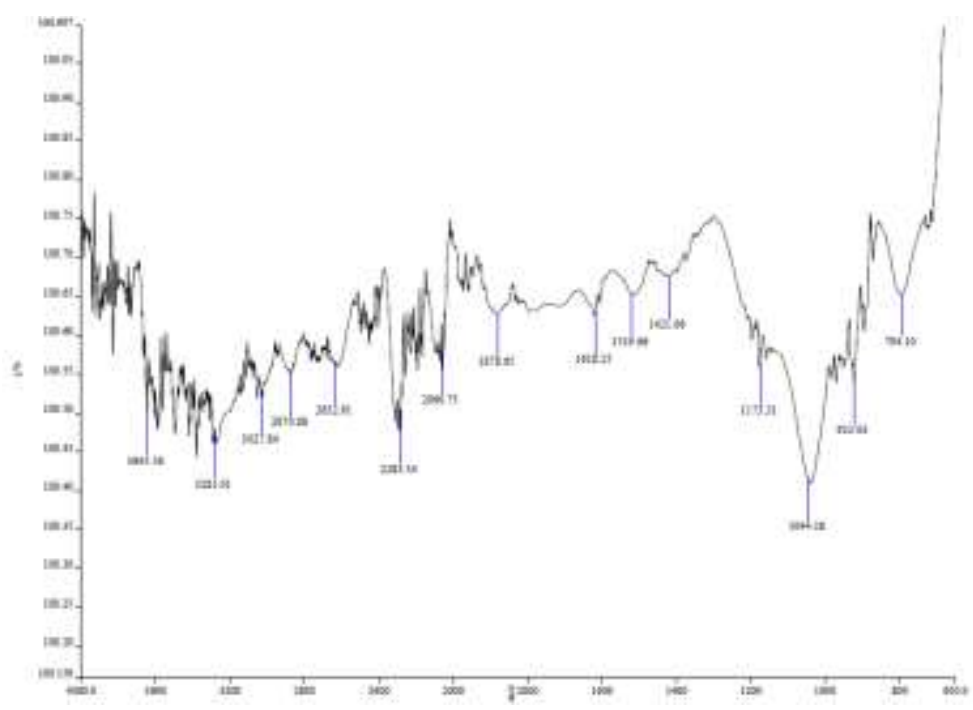

(b)

Figure 7. Moisture effect on layer (a) without and (b) with humidity 
The change in spectrum as shown in the plot is due to dipole-dipole interactions. The dissipation factor is in the order of $10^{-3}$ which shows the contamination free process suitable for sensor applications. Film quality is checked with the etch rate. Wet etching of zinc oxide layer is carried out using the wet chemistry consisting of $\mathrm{NH}_{4} \mathrm{Cl}: \mathrm{CH}_{3} \mathrm{COOH}: \mathrm{H}_{2} \mathrm{O}$ and etch rate achieved around 2.95um/min (Figure 8).

The dissipation factor indicates the quality of the film and lower value of the same commensurate with the deposition quality. The modified process results in one order change in the numerical value. The deposited structure is subjected to temperature cycling in chamber followed by optical inspection and adhesion tests.

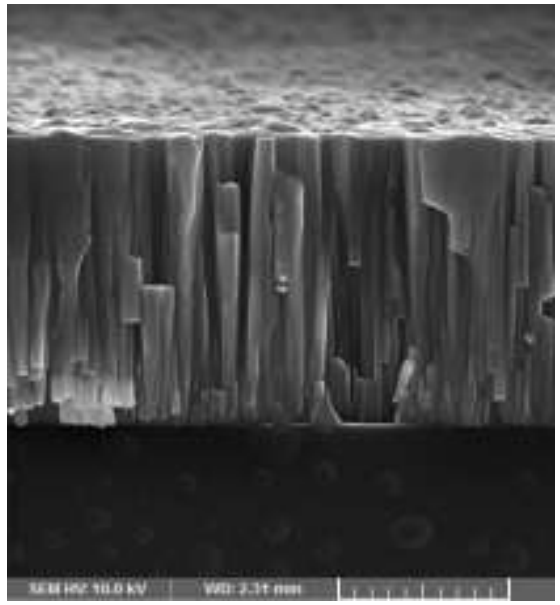

Figure 8. SEM plot after etching

The modified process can be utilized for the realization of MEMS based acoustic sensor. Annealing of the humidity exposed layer at $\mathrm{N}_{2}$ for 1 hour at $400^{\circ} \mathrm{C}$ resulting in the elimination of the Si-H bond. Piezo-electric properties of the deposited layer are analyzed by fabricating the acoustic device and characterizing its properties based on generation of charges due to acoustic vibrations and measuring its capacitance variation.

\section{Discussions}

Deposition of $\mathrm{ZnO}$ layer on silicon using sputtering techniques is carried out and characterized. This article details the characterization of the electrical and structural analysis of the ZnO film by various techniques such as XRD, FTIR, SEM, AFM. Calculated parameters of the deposited film are validated with the characterization results. Various measurement and characterization techniques are employed for the morphological study of the deposited film and further analyzed. Parameters such as wet etch rates and dissipation factor are important considerations for the piezo-electric based MEMS devices and the same is also discussed in the article. Film contamination can cause repeatability issues which are controlled in the present process and the same can be inferred with the dissipation factor. The deposited layer shows high resistivity and yield expected dielectric constant. The permittivity behaviour plot with the frequency shows the nearly constant value beyond $800 \mathrm{MHz}$ which can find application in RF devices. The deposition rate, growth temperature, gas composition, target impurity are important parameters for the thin film deposition and the same are optimized before implementation. The results show that $\mathrm{ZnO}$ film exhibits crystalline pattern and good homogeneity with the modified process. This process is validated with other trials such as humidity and measurement of dielectric constant in addition to temperature cycling resulting in achieving repeatable performance. This article brings out the comprehensive analysis of the various parameters and validation techniques for the deposited piezo-electric film. 


\section{Acknowledgement}

Authors acknowledge the support and guidance of Dr Ananth, Dr Gautum, Smt S. Abhram, Dr Z A Khan, Sh K Nagachenchaiah. Also gratefully acknowledge the support of colleagues at DOS, IISER and Delhi University.

\section{References}

[1]. Mahanth Prasad, V Shukla, V K Khanna," Design \& Fabrication of Si diaphgram ZnO piezo electric film based MEMS acoustic sensor using SOI wafers," IEEE Transactions on semiconductor manufacturing, Vol 26, No 2, May 2013, pp 233-236

[2]. K.Singh," Development of indigenous metal oxide gas sensors with embedded heater using CMOS process," Punjab Academy of Sciences Journal,13-14 (1 \& 2),pp 1-4,2015

[3]. Micro and Smart Systems, G K Ananthsuresh et al, Wiley India,2005

[4]. W.Huang," C-axis $\mathrm{ZnO}$ piezoelectric thin film prepared by RF magnetron sputtering for SAW filters," $2^{N D}$ International Conference on Computer Application and System Modelling, 2012,217-220

[5]. Zaheer, Nitesh \& Kamaljeet Singh," The challenge of convex corner undercutting phenomena observed in silicon micromachining and its circumvention," Technical Hindi seminar, SCL, Mohali 2016

[6]. K.Singh," Contaminations in MEMS Processes and Removal Methodology," ICTACT journal of microelectronics, Vol:3,Issue:1,pp 375-378,2017

[7]. U.Ozgur, Y I Alevov,C.Lui A. Teke, M A Reshchikov,S. Dogan, V Avrutin,S-J Cho, H. Morkeel," A comprehensive review of $\mathrm{ZnO}$ materials and devices," Journal of applied physics, 98, 2005

[8]. A. Arora, Anil Arora, V K Dwivedi, P J George, K. Srrnivas. V Gupta," Zinc oxide thin film based MEMS acoustic sensor with tunnel compensation," Sensors and Actuators, A141, 2008, pp 256-261

[9]. R. Ondo Nelong, H M omanda et al," Piezoelectric $\mathrm{ZnO}$ thin films by RF magnetron," The international journal of Engineering \& Sciences (IJES), Vol 2, Issue 12, pp 58-64,2013

[10]. D-L Cheng, K-S Kao, C-H Liang, Y-C Wang et al," Piezo electric response evaluation of $\mathrm{ZnO}$ thin film prepared by RF magnetron sputtering," $2^{\text {nd }}$ ICMSNT, MATEC web of conferences 109, 2017

[11]. X-G Xia, Y yuan, H Du, B Liu," Study of piezoelectric $\mathrm{ZnO}$ thin films for contact sensing and head actuation," IEEE transactions on magnetics, Vol 49, Issue 6, June 2013, pp 25392543

[12]. S D Shinde, G E Patil, D D Kayale, V G Wagh, V B Gailkwad, G H Jain," Effect of annealing on gas sensing performance of nano-structured $\mathrm{ZnO}$ thick film resistors," International Journal of smart sensing and intelligent systems, Vol 5, No 1, pp 277290,2012

[13]. K. Singh, A V Nirmal, "Annealing effects on stress behavior for PECVD deposited Oxide and Nitride thin layers for MEMS applications," AeCND 2018, Bengaluru, India. 